\title{
Evaluation of the Energy Impact on Indoor Small Cells and Backhaul
}

\author{
Albert Richard Moraes Lopes, Fabrício S. Farias, and João Crisóstomo Weyl Albuquerque Costa
}

\begin{abstract}
The use of heterogeneous networks, based on the macro and small cells composition, significantly reduces the power consumption in wireless networks. On the other hand, the implementation of the backhaul infrastructure responsible for ensuring communication and traffic support generated by the small cells causes a reverse regarding to the electric energy consumption. Thus, this study presents a model of predicting traffic for the next 15 years, from 2015 to 2030 , aiming to define the energy impacts of using small cells for indoor coverage. Furthermore, this study shows the discretized impact of each backhaul structure component in the growth of electric energy consumption.
\end{abstract}

Index Terms-Backhaul, energy efficiency, small cells, traffic model.

\section{INTRODUCTION}

Recently, the energy efficiency in mobile networks gained a great interest in the scientific community. In [1] it is shown that the mobile networks consume about $0.5 \%$ of the global energy, and the prospect is to double that consumption in the next five years. In [2] the authors suggest a strategy for deploying heterogeneous networks as a solution to reduce the total energy consumption of the network. This solution employs the use of small cells along with macro cells to improve the coverage and the network experience of the user. The macro cells better serve the outdoor users but have difficulties with indoor users [3], which according to [4], it represents approximately $70 \%$ of all mobile traffic.

The use of small cells, such as femtocells, in indoor environment meets in an efficient way the users of that environment [3]. However, in the studies [2], [5] and [6], it was found that in heterogeneous networks the backhaul represents a significant portion of the energy consumption being pointed as a possible bottleneck for the growth of the heterogeneous network. Therefore, the backhaul cannot be neglected as the heterogeneous network is popularized. Thus, currently the total energy consumption for heterogeneous mobile networks has been considered as the sum of the consumption generated by the wireless part and the backhaul segment.

Manuscript received November 10, 2014; revised March 4, 2015.

Albert Richard Moraes Lopes is with the Laboratory of Applied Electromagnetics, Federal University of Pará, Brazil (e-mail: albert.richard@gmail.com).

Fabrício S. Farias is with the Laboratory of Applied Electromagnetics in Federal University of Pará. He is also with UFPA Campus Cametá, Brazil (e-mail: fabriciosf@ufpa.br).

João Crisóstomo Weyl Albuquerque Costa is with Electrical and Computer Engineering, Federal University of Pará, Brazil (e-mail: jweyl@ufpa.br).
In order to reduce the energy consumption of backhaul, a few studies examined the impacts of possible backhaul infrastructures for heterogeneous mobile networks. In [1] the backhaul technologies and microwave fiber were analyzed. This study considered an urban scenario composed of a heterogeneous mobile network (composed of macro cells and pico cells) or homogeneous (consisting only of macro cells). The results reinforce that adopting heterogeneous mobile networks reduce the total energy consumption and increase the energy consumption in the backhaul segment. Moreover, it showed that using different types of backhaul segment technology can change the power consumption. During the study, it was concluded that the use of fiber significantly reduces the energy consumption. However, the model mobile network presented considered only outdoor traffic, assuming that the traffic is equal to the maximum network capacity. Due to this consideration of traffic, the backhaul infrastructure rapidly increases over the years. Furthermore, this study examined the backhaul segment homogeneously, disregarding technologies based on copper, as VDSL2, which in entrepreneurial studies, as in [7], point to the survival of this technology for a few more years.

Meanwhile in [5], the authors analyzed the backhaul homogeneously, only microwave, and heterogeneous, fiber + copper and fiber + microwave, under the influence of heterogeneous mobile network for indoor and outdoor environment, covered by femtocells and macrocells respectively. In addition, it was used the model for large-scale and long-term presented in [8], to determine the growth of the heterogeneous mobile network. With this study, it was concluded that the backhaul heterogeneous option, composed of fiber technologies - covering indoor environment, and microwave - covering outdoor environment, is a promising candidate in scenarios with high penetration rate of femetocells base stations (BSs).

After the reference review, it was found two caveats that limit the results in the literature. Such limitation opens possibilities for improvements aiming to approximate the results of current scenarios.

The first caveat is to consider the analysis of electrical energy consumption considering all the indoor and outdoor environments. Each environment has its own behaviors and analysis, in other words, in a given environment the technology can be discarded, but in another environment it can be valuable. For example, copper is not suitable for outdoor 4G, but it can still play an important role in indoor infrastructure providing up to $100 \mathrm{Mbps}$ to 300 meters [9], [10]. As mentioned previously, indoor users represent a larger share of the traffic, and they connect the indoor networks through opportunistic algorithms that control, for example, 
the best signal choice, and transmission capacity [11].

The second caveat is to assess the backhaul as one segment only. So that, it is possible to identify that only the backhaul is a bottleneck but not identify where the biggest problem of power consumption in the structure of backhaul is located.

The objective of this paper is to present a predicting traffic model for the period of 2015-2030. Provide a distribution planning of BSs according to the coverage area and investigate an efficient way to provide coverage through small cells to indoor mobile users respecting the growth of the backhaul power consumption.

The remainder of this study is presented as follows: In Section II, the methodology used is detailed. In Section III, it is presented the mathematical modeling treating models such as the predictive traffic and backhaul energy consumption. Section IV shows the scenario and parameter settings used, and Section V summarizes and discusses the results obtained. Finally, conclusions are presented in Section VI.

\section{Methodology}

This study is divided into the following steps: traffic forecasting; city and mobile network backhaul infrastructure sizing; and energy consumption assessment.

In the first step, it was created an average traffic demand estimative per area taking into consideration the type of the scenario, in other words, urban, suburban or rural areas. As input parameters, there are the following inputs: average rate of data consumption per subscriber, subscriber density as a function of time, diversity of terminal areas (dense urban, urban, rural, etc.) and environments (indoor and outdoor). In order to obtain the variables, it was used the following parameters: population growth, demand required per user per terminal and amount of signatures per person. These parameters were obtained by exponential or linear prediction model, more details in Subsection III-A.

The second step consists of sizing the city. In this phase, the city is set using the parameterization of the following inputs: the number of blocks, buildings and apartments. The parameterization is conducted as a function of the size of each zone, in other words, urban, suburban or rural. The details of this phase are presented in Subsection III-B.

The third step consists in the phase of sizing the mobile network. In this phase, the number of base stations required is determined to meet the demand and network coverage. The number of base stations is calculated as a function of the traffic forecast scenario result, obtained in the previous step, and the size of the city. The details of this phase are shown in Subsection III-C.

The Fourth step is the phase of sizing the backhaul. This phase determines the amount of the equipment that composes the backhaul architectures. The backhaul sizing is as a function of the wireless network, which is dimensioned in the previous step, and the access technology type such as fiber optics, microwave, copper or hybrid. This phase is detailed in the subsections of Subsection III-D.

Finally, the last step is the evaluation of the energy consumption impact on the backhaul. In the Subsection III-D, it is presented the generic model of the backhaul energy consumption and in its subsections are presented the consumption by type of backhaul architecture depending on the means of access technology.

\section{MODELING}

\section{A. Traffic Forecast}

It was considered the traffic forecast model, which was based on the long-term and large-scale model defined in [8], in order to estimate the European average traffic demand over the years 2015 to 2030 . The total traffic generated $R(t)$ in $\mathrm{Mbps} / \mathrm{km}^{2}$ to a city is defined by equation 1 .

$$
R(t)=\sum_{k} \sum_{z} \sum_{e} r(t, k) s(t, k, z, e)
$$

where " $t$ ", " $k$ ", “ $z$ " and " $e$ " represent the time in years, number of terminal types, zones and environments, respectively. $r(t, k)$, given in Mbps/terminal, represents the average data rate per user at any given time " $t$ " and by the terminal type " $k$ ". On the other hand $s(t, k, z, e)$ represents the subscribers density as a function of time, terminal type, zone and environment. Unlike [8], it was ignored the fact that the total traffic is served by a number of operators in a given area.

In this modeling, three terminal types are considered: Mobile PC, tablet and Smartphone. Initially, it was consider that a mobile PC generates twice and eight times more traffic than a tablet and smartphone, respectively. Moreover, the users are divided into two groups (i.e, "heavy" and "ordinary users") according to the ability required by the user. The ability required by an "ordinary user" is equivalent to $1 / 8$ of the capacity required by a "heavy user". The average daily demand for terminal $k$ is defined in [8] as:

$$
r(t, k)=\frac{h(t) r(t, k)^{h e a v y}+[100-h(t)] r(t, k)^{\text {ordinary }}}{100}
$$

Assuming that $h(t)$ is the subscribers percentage who are "heavy user" for a given time $t$. In [8], $h(t)$ varies from $10 \%$ to $30 \%$ between the 2015 and 2030 . Considering that $h(t)$ grows linearly in the meantime, $h(t)$ can be express by equation 3 .

$$
h(t)=\frac{t}{75}-26.767
$$

Here $r(t, k)^{\text {heavy }}$ and $r(t, k)^{\text {ordinary }}$ represents the average data rate at peak times in Mbps for "heavy" and "ordinary user", respectively. $r(t, k)^{\text {heavy }}$ is calculated by Equation 4 .

$$
r(t, k)^{h e a v y}=r\left(t_{0}, k\right)^{h e a v y}\left(1+r_{t x}(k)\right)^{\left(t-t_{0}\right)}
$$

$t_{0}$ is the initial time of the study, which in this case study is 2015. In [8], the $r\left(t_{0}, k\right)^{\text {heavy }}$ is equals to $2 \mathrm{Mbps}, 1 \mathrm{Mbps}$ and $0.25 \mathrm{Mbps}$ for mobile PC, tablet and Smartphone, 
respectively. Furthermore, $r_{t x}(k)$ is the growth rate of the $r\left(t_{0}, k\right)^{\text {heavy }}$ annual for the terminal " $k$ ". In [3], the $r_{t x}(k)$ is equal to $25 \%, 30 \%$ and $25 \%$ of annual growth for mobile PC, tablet and smartphone, respectively. After calculating the $r(t, k)$, it remains only to calculate the subscribers density in order to obtain $R(t) \cdot s(t, k, z, e)$ in teminal $/ \mathrm{km}^{2}$, is given by equation 5 .

$$
s(t, k, z, e)=\frac{\eta(t, k, z, e)}{A(z, e)}
$$

$A(z, e)$ is the area of the city which refers to the environment " $m$ " of the zone " $z$ ". Moreover, $\eta(t, k, z, e)$, is the terminal " $k$ " amount located in the environment " $e$ " of the zone " $z$ " for a given time $t$, which is given by equation 6 .

$$
\eta(t, k, z, e)=\alpha(T) \gamma(k) \phi(z) \beta(e) p(t) s_{p}(t)
$$

$\alpha(T)$ represents the daily variation percentage of active users over time through $\mathrm{T}$, in hours. It is know that, the real interest is in testing the limit, it was considered $\alpha(T)$ as a constant equals to $\alpha_{\max }$, given in [8], i.e., $16 \%$ of subscribers will be active. $\gamma(k), \varphi(z)$ and $\beta(e)$ represent the percentual of signatures per terminal " $k$ ", zone " $z$ " and environment " $e$ " respectively. $\gamma(k)$ is equal to $25 \%, 5 \%$ and $50 \%$ for mobile PC, tablet and Smartphone, respectively, according to [8]. $p(t)$ and $s_{p}(t)$ represent the population and the number of subscriptions per person for a given time $t$. They are obtained by equations 7 and 8 .

$$
\begin{gathered}
p(t)=p\left(t_{0}\right)\left(1+t x_{p}\right)^{\left(t-t_{0}\right)} \\
s_{p}(t)=s_{p}\left(t_{0}\right)+g_{s_{p}}\left(t-t_{0}\right)
\end{gathered}
$$

$t x_{p}$ and $g_{s_{p}}$ are the population the subscriptions growth per person, respectively. In [12] and [13], $t x_{p}$ and $g_{s_{p}}$ are equal to $0.7 \%$ and approximately 0.0314 of annual growth, respectively. $s_{p}\left(t_{0}\right)$ is the initial signatures amount per habitant. In [13], as $s_{p}$ for 2011 is equals to 1.19 signatures per person and using equation (7) it was obtained $s_{p}\left(t_{0}\right)$ equals to 1.3156. Furthermore, $p\left(t_{0}\right)$ is the initial population given by equation 9 .

$$
p\left(t_{0}\right)=\sum_{z} \rho(z) A(z)
$$

$\rho(z)$ is the population density per city area. In [8], $\rho(z)$ for "dense urban", "urban", "suburban", "rural" and "wild" zones are equals to $3000,1000,500,100$ and 25 persons per $\mathrm{km}^{2}$, respectively. $A(z)$ is the total area for the zone " $z$ ".

\section{B. City Sizing}

Consider a city with the area $A$ in $\mathrm{km}^{2}$ is subdivided into " $z$ " zones with population density $\rho(z)$. Each " $z$ " zone is divided into " $e$ " environments possessing blocks with identical dimensions regardless of zone. The amount of blocks, blocks for buildings, floors per building and apartments per floor are represented by $N_{b}, N_{b p b}, N_{f p b}$ and $N_{a p f}$, respectively. These variables are as a function of " $z$ " zone. Furthermore, $N_{b}(z)$ is obtained dividing the zone area by the area of the block, which are represented by $A(z)$ and $A_{\text {block }}$, respectively. After the given considerations about these variables, the number of apartments per zone " $z$ " is given by

$$
N_{\text {apartments }}(z)=N_{b}(z) \times N_{b p b}(z) \times N_{f p b}(z) \times N_{a p f}(z)
$$

\section{Indoor Wireless Network Sizing}

For the indoor wireless network sizing, it was only considered the implementation of conventional Femtocells base stations (FemtoC) or Femtocells Wireless over Cable (FemtoWoC). Each FemtoC is a standalone device configuring a BS. In [14], the FemtoWoC is presented as a solution for indoor BS compared to FemtoC. The FemtoWoC consists of a A/A Converter and Multi-Cell and BS (McBS). For this study, it was considered that the A/A Converter as a FemtoWoC BS. Knowing that, it was considered that each FemtoC and FemtoWoC is implemented for only one BS per apartment and one per building floor, respectively. Therefore, the equation 10 represents the maximum amount of FemtoC BS that can be implemented, which is equal to the number of apartmens in zone " $z$ ". Since equation 11 is the maximum amount of FemtoWoC, which is given as a function of the apartments quantity and apartments per floor of the building in the zone " $z$ ". From this, the amount of BS to be implemented in the city is given by equation 12 .

$$
\begin{gathered}
N_{\max }(z, \text { FemtoC })=N_{\text {apartments }}(z) \\
N_{\max }(z, \text { FemtoWoC })=\left(\frac{N_{\text {apartments }}(z)}{N_{\text {apf }}(z)}\right) \\
B S(t, b)=\sum_{z}\left\lceil\min \left(\frac{R(t, z)}{C_{d l}(b)}, N_{\max }(z, b)\right)\right]
\end{gathered}
$$

$R(t, z)$ is the traffic generated by the zone " $z$ ", which can be obtained by equation 1 . And " $b$ " is the kind of BS indoor. On the other hand, $C_{d l}(b)$ is the maximum downlink capacity for BS "b".

\section{Total Energy Consumption in Backhaul}

In [15], it is proposed the generic backhaul model composed by three segments: "cell site (CS)", "hub site (HS)" 
and "Central Office (CO)". The "CS" has the function of aggregating traffic from one or more BSs. On the other hand, the "HS" works aggregating traffic from one or more "CS". And the "Central Office" aggregates the traffic of one or more "hubs". Depending on the technology type used in each location, the communication between these segments uses particular means of access that will qualify the backhaul type. Among the most common ways are fiber optics, microwave and copper.

Based on this generic architecture, the total energy consumption in "Watt" for a given backhaul architecture is provided by equation 13 . It was considered that the aggregate functions, commutation and routing are ideally performed in order to transport the traffic between the network core and the network access.

$$
\begin{aligned}
P(t, a)= & P_{C S}(t, a)+P_{H S}(t, a) \\
& +P_{C O}(t, a)
\end{aligned}
$$

$P_{C S}(t, a), P_{H S}(t, a)$ and $P_{C O}(t, a)$ represent the total energy consumption by "CS", "HS" and "Central Office" for backhaul architecture " $a$ ". Considering the use of FemtoC and FemtoWoC BSs and the means of access, such as fiber and copper, it is presented four types of backhaul architecture, where three types are for mobile network composed of FemtoCs and one type composed of FemtoWoC. The architectures are explained in the following subsections, and illustrated in Fig. 1.

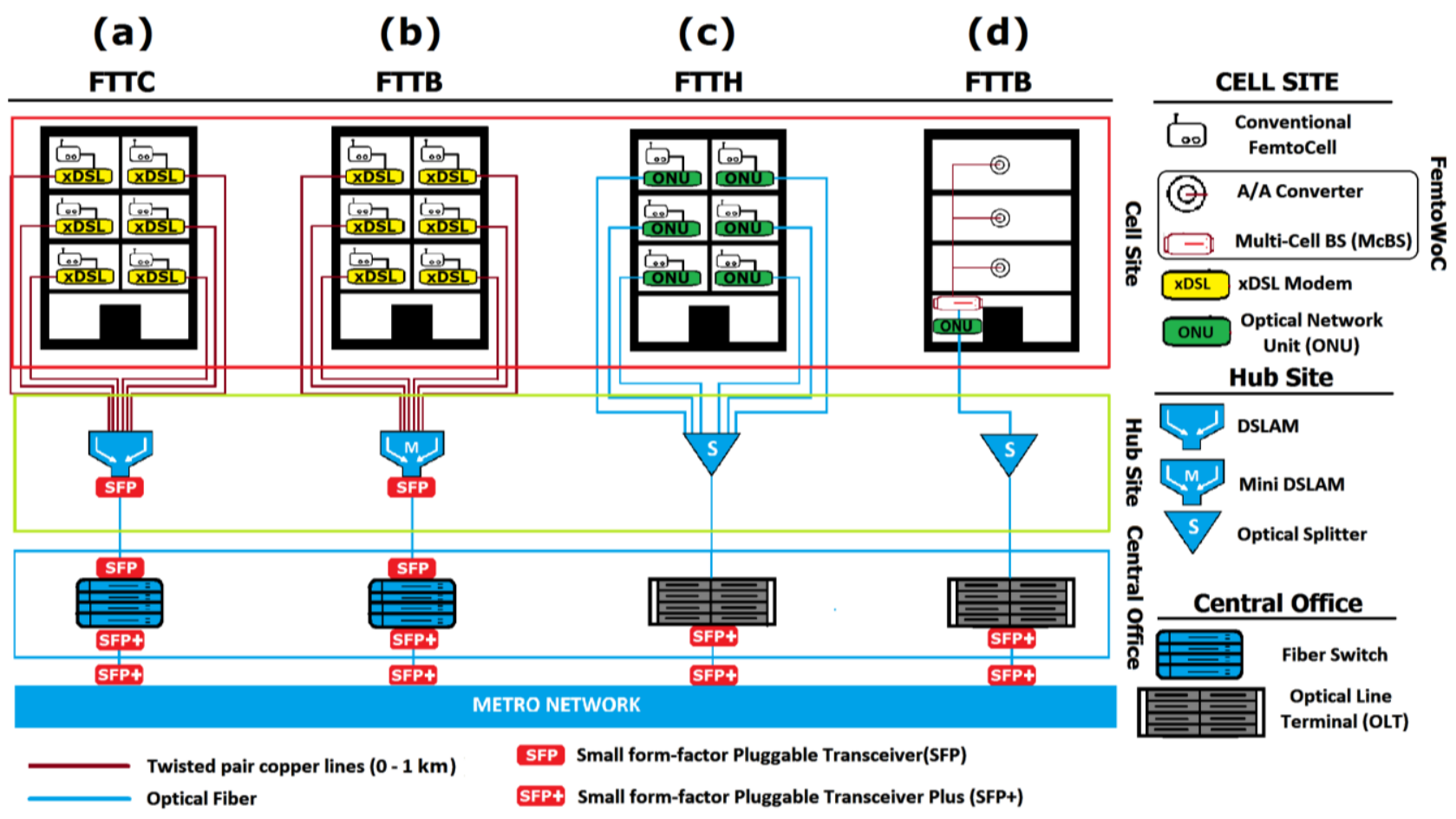

Fig. 1. Backhaul architectures.

\section{1) Architecture 1 (Arc1): Fiber-to-the-curb/cabinet with FemtoC BS}

The backhaul solution Fiber-to-the-Curb/Cabinet (FTTC) for FemtoC presented in Fig. 1 (a) is a hybrid architecture that employs the use of fiber and copper. In this architecture the Femto BS traffic is "backhauled" for a XDSL modem in "CS". In this study, it was considered the modem type "Very-high-speed Digital Subscriber Line 2" (VDSL2), which provides high-speed connections over twisted pair of copper cables. Each modem is connected to the DSLAM in "HS" through a copper connection. The DSLAM is stored in a cabinet in the street next to the users. The choice for VDSL2 was due to maximum downlink rate achieved that meet the backhaul criteria for Femto over other xDSL technology. According to [16] and [7], the VDSL2 reaches up to 100 Mbps for distances of $300 \mathrm{~m}$ and $1.5 \mathrm{~km}$, under certain conditions. Due to this VDSL2 limitation, it is better for the DSLAM to be distant from the user at a maximum are of 300 m. DSLAMs are connected to "Fiber Switches" (SFs) in the "Central Office" by 1 Gbps fiber links point-to-point. In order to transmit and receive the optical signal, it is used small form-factor plugglable transceivers (SFPs). SFs aggregate traffic of the entire wireless network before sending to the metro network (MN)" via 10Gbps fiber links and SFP+ modules. As it was shown in Equation 13, the total backhaul power consumption is the sum of the CS, HS and CO power consumption, which for this architecture are given by equations 14,15 and 16 :

$$
\begin{gathered}
P_{C S}(t, \operatorname{arc} 1)=B S(t, \text { Femto } C) \times P_{\text {modem }} \\
P_{H S}(t, \operatorname{arc} 1)=N_{D S L A M}(t, \operatorname{arc} 1) \\
\times\left(P_{D S L A M}+2 \times P_{S F P}\right) \\
P_{C O}(t, \operatorname{arc} 1)=N_{f s}(t, \operatorname{arc} 1) \times\left(P_{f s}+2 \times P_{S F P+}\right)
\end{gathered}
$$

where $P_{\text {modem }}, P_{D S L A M}, P_{f s}, P_{S F P}$ and $P_{S F P+}$ represent the energy consumed in "Watt" by the VDSL2 and DSLAM modem; 
"fiber switch"; SFP and SFP+, respectively. Moreover, $N_{D S L A M}(t, a r c 1)$ and $N_{f s}(t, \operatorname{arcl})$ represent the number of DSLAM and "fiber switch" for the backhaul FTTC at the time "t". $N_{D S L A M}(t, \operatorname{arc} 1)$ is as a function of the number of ports in used by the DSLAM: $N_{D S L A M}(t$, arc 1$)=\left\lceil\frac{B S(t, \text { Femto } C)}{N_{D_{\text {DSLAM }} \text { ports }}}\right\rceil$. Similarly, $N_{F S}(t, a r c 1)$ is based as a function of ports used by the FS:

$N_{f s}(t, \operatorname{arc} 1)=\left\lceil\frac{N_{D S L A M}(t, \operatorname{arc} 1)}{N_{f_{\text {ports }}}}\right\rceil$.

2) Architecture 2 (Arc2): Fiber-to-the-building with FemtoC BS

The backhaul solution Fiber-to-the-building (FTTB) for FemtoC BS, represented in Fig. 1 (b), is also a hybrid architecture that employs fiber and copper. This architecture is similar Arc 1. The only difference is the DSLAM replacement for the miniDSLAM in "HS". In this case the "HS" is within each building instead of booths on the streets. After the "HS" the Arc 2 is similar to Arc 1. The power consumption of the segments in "Watt" for this architecture is obtained by equations 17,18 and 19 .

$$
\begin{gathered}
P_{C S}(t, \operatorname{arc} 2)=B S(t, \text { Femto } C) \times P_{\text {modem }} \\
P_{H S}(t, \operatorname{arc} 2)=N_{\text {MiniDSLAM }}(t, \operatorname{arc} 2) \\
\times\left(P_{\text {MiniDSLAM }}+2 \times P_{S F P}\right) \\
P_{C O}(t, \operatorname{arc} 2)=N_{F S}(t, \operatorname{arc} 2) \times\left(P_{f s}+2 \times P_{S F P+}\right)
\end{gathered}
$$

where $P_{\text {MiniDSLAM }}$ represent the energy consumed in "Watt" for miniDSLAM. Moreover, the $N_{\text {MiniDSLAM }}(t, \operatorname{arc} 2)$ and $N_{f s}(t, a r c 2)$ represent the amount of miniDSLAM and Fiber Switch for the backhaul FTTB in the time " $t$ ". $N_{\text {MiniDSLAM }}(t, \operatorname{arc} 2)$ is as a function of ports using the

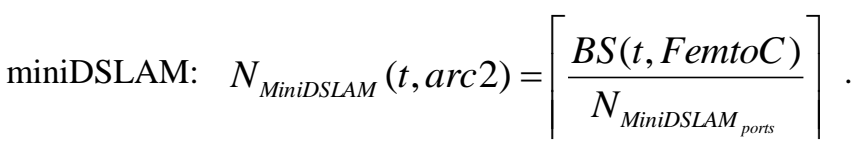
Similarly, the $N_{f s}(t, \operatorname{arc} 2)$ is as a function of ports using FS:

$$
N_{f s}(t, \operatorname{arc} 2)=\left\lceil\frac{N_{\text {MiniDSLAM }}(t, \operatorname{arc} 2)}{N_{f s_{\text {ports }}}}\right\rceil \text {. }
$$

\section{3) Architecture 3 (Arc3): Fiber-to-the-home with}

\section{FemtoC BS}

The backhaul solution Fiber-to-the-Home (FTTH) with FemtoC BS, presented in Fig. 1 (c), is architecture with means of access only through fiber. As with other architectures, each FemtoC is in one apartment. The difference is that the equipment will "backhauled" the traffic in the "CS" is the "Optical Network Unit" (ONU). The aggregate traffic at the ONUs is drained to the "Optical Splitter" via fiber cables in "HS". Each "Optical Splitter" is connected via optical fiber to a "Optical Line Terminal" (OLT) port at the CO. The OLTs add all network traffic before sending them to the $\mathrm{MN}$ via fiber links of $10 \mathrm{Gbps}$ in SFP+ modules. The energy consumption in "Watt" is given by the sum of the parts of this architecture that are obtained by equations 20, 21 and 22 .

$$
\begin{gathered}
P_{C S}(t, \operatorname{arc} 3)=B S(t, \text { Femto } C) \times P_{\text {onu }} \\
P_{H S}(t, \operatorname{arc} 3)=N_{\text {splitter }}(t, \operatorname{arc} 3) \times P_{\text {splitter }} \\
P_{C O}(t, \operatorname{arc} 3)=N_{O L T}(t, \operatorname{arc} 3) \times\left(P_{O L T}+2 \times P_{S F P+}\right)
\end{gathered}
$$

where $P_{\text {onu }}, P_{\text {splitter }}, P_{O L T}$ and $P_{S F P+}$ represent the power consumed by the ONU, "Optical Splitter", OLT and SFP+, respectively. $P_{\text {splitter }}$ is the passive type, so it does not consume energy. Furthermore, $\quad N_{\text {splitter }}(t, \operatorname{arc3}) \quad \mathrm{e}$ $N_{\text {OLT }}(t, \operatorname{arc} 3)$ represents the amount of "Optical Splitter" and OLT for the backhaul FTTH at time " $\mathrm{t}$ ". $N_{\text {splitter }}(t, \operatorname{arc3})$ is based on the number of "Optical Splitter" ports: $N_{\text {splitter }}(t, \operatorname{arc} 3)=\left\lceil\frac{B S(t, \text { FemtoC })}{N_{\text {splitter }} \text { ports }}\right] \quad$. Similarly $N_{\text {OLT }}(t, \operatorname{arc} 3)$ is based on the number of OLT ports: $N_{\text {OLT }}(t, \operatorname{arc} 3)=\left\lceil\frac{N_{\text {splitter }(t, \operatorname{arc} 3)}}{N_{O L T_{\text {port }}}}\right\rceil$.

\section{4) Architecture 4 (Arc4): Fiber-to-the-building with FemtoWoC BS}

The Fiber-to-the-building (FTTB) solution for FemtoWoC BS, presented in Fig. 1 (d), is hybrid architecture composite of fiber and copper. Each FemtoWoC is implemented in each floor of the building and its traffic is aggregated in the McBS in "CS". They are connected by twisted pair copper cables. Each McBSs is connected to a "ONU", which is also the leased in the "CS" for fiber cable. The aggregate traffic at the ONUs is drained to the "Optical Splitter" in "HS" via fiber cables. From the "Optical Splitter" connections and equipment are the same used in FTTH. The energy consumption in "Watt" of this architecture segments is obtained by equations 23,24 and 25 .

$$
\begin{gathered}
P_{C S}(t, \operatorname{arc} 4)=N_{M c B S} \times P_{M c B S} \\
P_{H S}(t, \operatorname{arc} 4)=N_{\text {splitter }}(t, \operatorname{arc} 4) \times P_{\text {splitter }} \\
P_{C O}(t, \operatorname{arc} 4)=N_{O L T}(t, \operatorname{arc} 4) \times\left(P_{O L T}+2 \times P_{S F P+}\right)
\end{gathered}
$$

where $P_{M C B S}, P_{s p l i t t e r}, P_{O L T}$ and $P_{S F P+}$ represent the energy consumed by the "McBS", "Optical Splitter", OLT and SFP +, respectively. $P_{\text {splitter }}$ is the passive type, so it does not consume energy. Furthermore, $N_{M c B S}, N_{\text {splitter }}(t, \operatorname{arc} 4)$ and $N_{\text {OLT }}(t, \operatorname{arc} 4)$ represent McBS quantity, "Optical Splitter" 
and OLT for the backhaul FTTB in time " $\mathrm{t}$ ". $N_{M c B S}$ is a function of the number of MCBs ports and the amount of FemtoWoC: $N_{M c B S}(t)=\left[\frac{B S(t, \text { FemtoWoC })}{N_{M c B S_{\text {ports }}}}\right]$.

$$
N_{\text {splitter }}(t, \operatorname{arc} 4)=\left\lceil\frac{B S(t, \text { FemtoC })}{N_{\text {splitter }} \text { ports }}\right] \text { is based on the }
$$

number of "Optical Splitter" ports. Similarly, $N_{O L T}(t, \operatorname{arc} 4)$ is based on the number of OLT ports: $N_{\text {OLT }}(t, \operatorname{arc} 4)=\left\lceil\frac{N_{\text {splitter }}(t, \operatorname{arc} 4)}{N_{O L T_{\text {pors }}}}\right\rceil$.

\section{StUdy CASE - PARAMETERS DEFINITION}

In this section, it is presented the assumptions used and the case study. Then, it is explained the methodology used to estimate the total energy consumption of the network.

For this study, it was adopted the scenario of city with urban and dense urban areas organized as the Manhattan city model, as shown in Fig. 2. For both cases, it was studied indoor environments, in other words, it was analyzed only traffic inside buildings. Zone dependent variables " $z$ " are given in Table I.

TABLE I: CITY DATA CONFIGURATION

\begin{tabular}{ccc}
\hline \hline Variable & Urban & Dense Urban \\
\hline$N_{b p b}$ & 2 & 4 \\
$N_{f p b}$ & 2 & 3 \\
$N_{a p f}$ & 2 & 2 \\
\hline \hline
\end{tabular}

\begin{tabular}{|c|c|c|}
\hline Variable & Urban & Dense Urban \\
\hline$N_{\text {apartments }}(z)$ & 69966 & 86400 \\
\hline$\rho(z)$ & 1000 & 3000 \\
\hline$\varphi(z)$ & $25 \%$ & $75 \%$ \\
\hline Power Consumption Parameters & & Value \\
\hline$P_{D S L A M} / N_{D S L A M}$ & & $64 \mathrm{~W} / 16$ ports \\
\hline$P_{f s} / N_{f_{\text {ports }}}$ & & $300 \mathrm{~W} / 24$ ports \\
\hline$P_{\text {splitter }} / N_{\text {splitter }_{\text {ports }}}$ & & $0 \mathrm{~W} / 16$ ports \\
\hline$P_{\text {MiniDSLAM }} / N_{\text {MiniDSLAM }_{\text {ports }}}$ & & $64 \mathrm{~W} / 48$ ports \\
\hline$P_{O L T} / N_{O L T_{p o r t s}}$ & & $300 \mathrm{~W} / 40$ ports \\
\hline$P_{M c B S} / N_{M c B S_{p o r t s}}$ & & $45 \mathrm{~W} / 16$ ports \\
\hline$P_{\text {Modem }} / P_{O N U}$ & & $10 \mathrm{~W} / 5$ ports \\
\hline$P_{S F P} / P_{S F P+}$ & & $2.5 \mathrm{~W} / 3$ ports \\
\hline Base Station & & Downlink Capacity \\
\hline $\begin{array}{l}\text { FemtoC } \\
\text { FemtoWoC }\end{array}$ & & $\begin{array}{l}50 \mathrm{Mbps} \\
50 \mathrm{Mbps}\end{array}$ \\
\hline
\end{tabular}

The users are exclusively served by small cells BS, as shown in Fig. 1. It was used FemtoC and FemtoWoC base stations and as a small cells possibility. The small cells are placed the apartments or floors building operating as a licensed spectrum, focusing on the demand for coverage and capacity for indoor users. It was consider that the average data rate per user changes over the years (Subsection III-A).

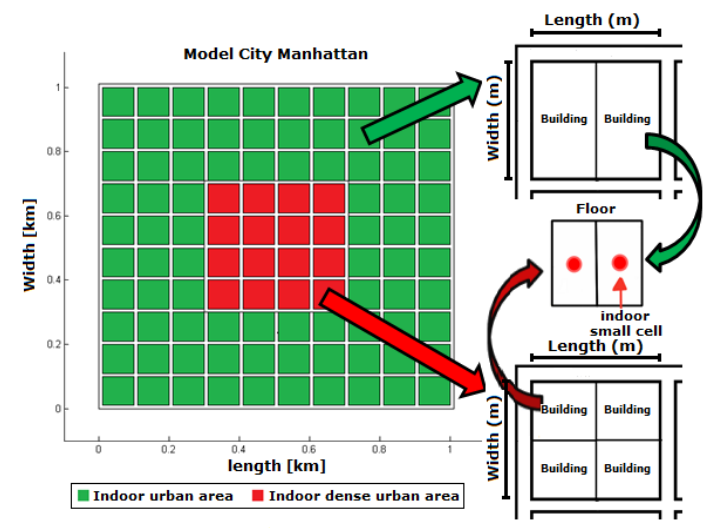

Fig. 2. City data configuration.

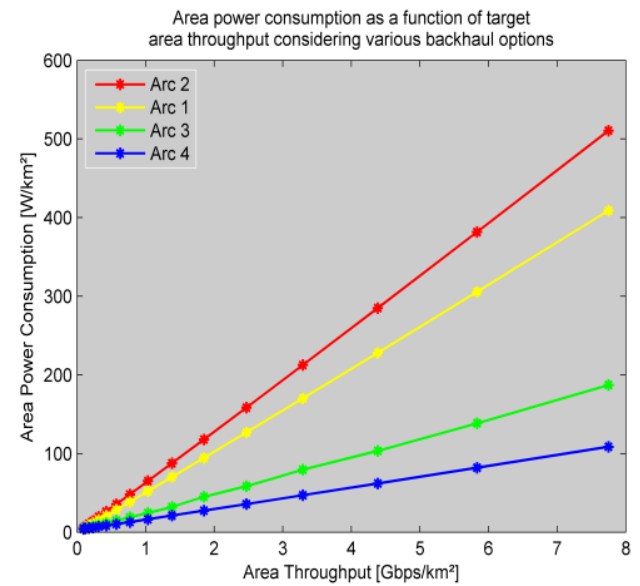

Fig. 3. Energy consumption per area of the backhaul architecture as function of the throughput per area.

For this case study, it was considered an average European city like Stockholm, with an area of $10 \mathrm{~km} \times 10 \mathrm{~km}$, and the blocks with dimensions of $100 \mathrm{~m} \times 100 \mathrm{~m}$. The city has two areas: urban and dense urban. Considerations about zone dependent variables are presented in Table I and others in the Section III. In this study, it was only considered indoor environment. In [4], the indoor traffic environment corresponds for $70 \%$ of all traffic. The terminals distribution is $20 \%, 5 \%$ and $50 \%$ for mobile PC, Tablet and Smartphone. Using these data and the previously mentioned in Section III, it was calculate the average traffic demand at peak times using the equations presented in Subsection III-A. It was assumed that the demand for the indoor user is only served by small cells (FemtoC or FemtoWoC). In this scenario, the traffic generated by the indoor environment is assumed to be added to the "MN" through four backhaul architectures presented in Subsection III-D. The parameters for the backhaul architectures and the equipment energy consumption are listed in Table II.

\section{Study CASE - Results}

Fig. 3 shows the energy consumption per area, the four backhaul architectures as function of the backhaul throughput per area. Recalling that Arc 1, Arc 2 and Arc 3 architectures 
FTTC, FTTB and FTTH with FemtoC BS, respectively, and Arc 4 is the FTTB with FemtoWoC BS. It was noted that, in all backhaul architectures the power consumption increases linearly with the throughput increase. Comparing the architectures with Femto BS, the power consumption decreases from Arc 2, Arc 1, Arc 3, following that order. Therefore, the most and the least efficient in terms of energy is FTTH and FTTB.

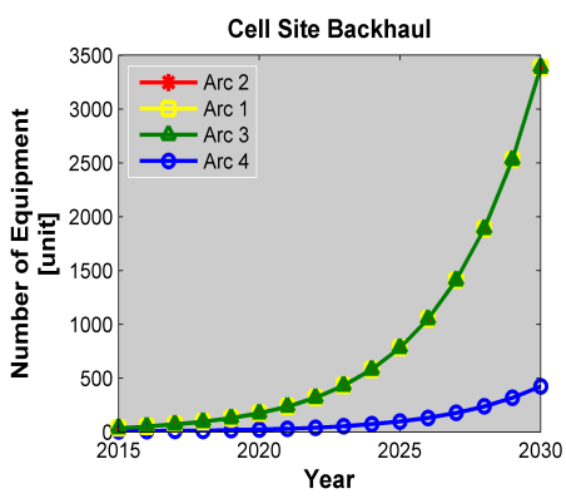

(A)

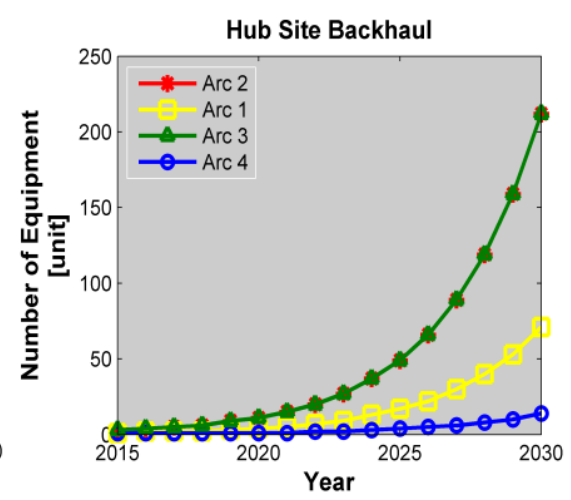

(B)

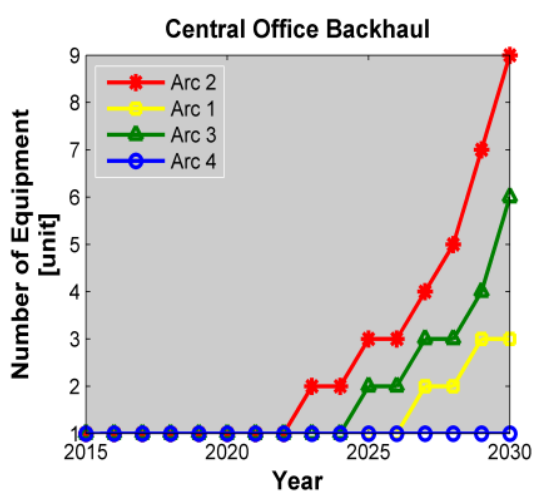

(C)

Fig. 4. Device amount per backhaul segment over time.

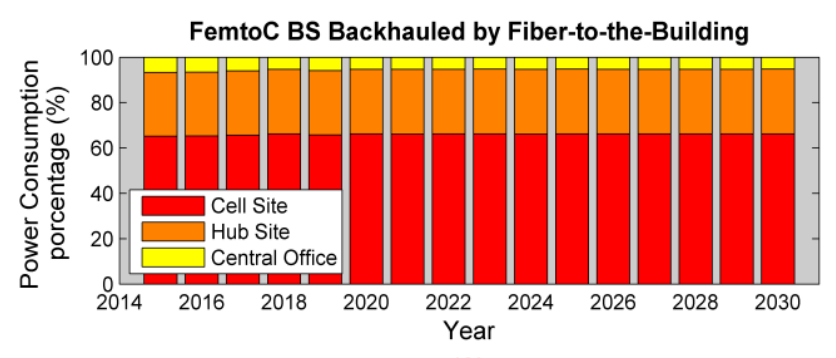

(A)

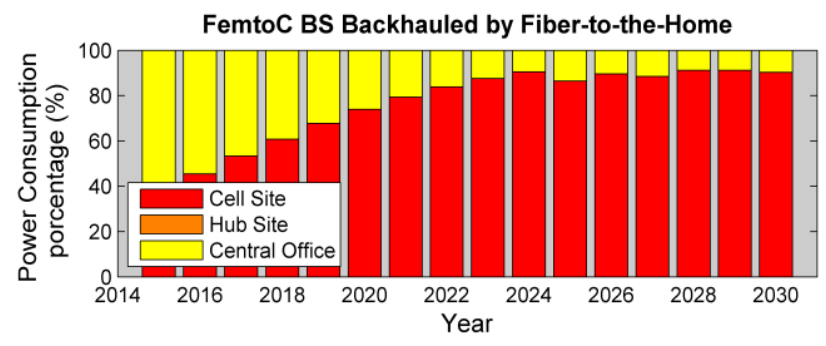

(C)

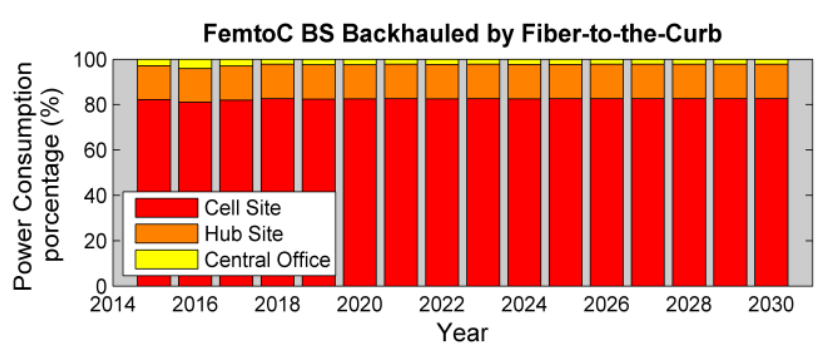

(B)

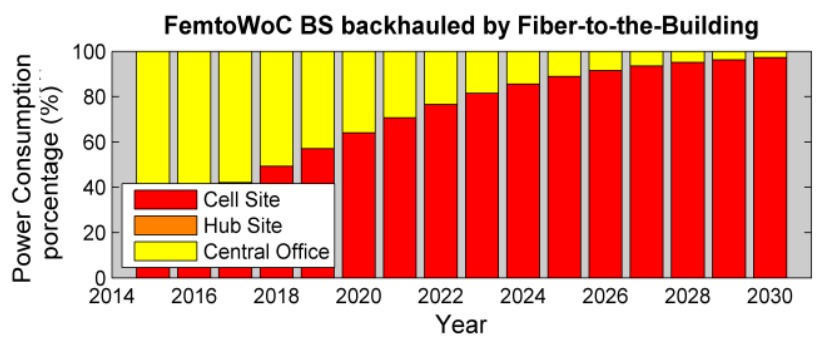

(D)

Fig. 5. Power consumption percentage of the backhaul segments relative to the backhaul total power consumption.

Considering only the Arc 2 and Arc 1 architectures, using VDSL2 technology, it was note that the FTTB consumes more energy than the FTTC. The reason is shown in Fig. 4. The device amount in the HS and CO is higher in the Arc 2 than in the Arc 1 due to the own backhaul configuration. The miniDSLAMs and DSLAMs are implemented by building and set of buildings, respectively. The greater is the number of devices in HS, it directly impacts the device amount of $\mathrm{CO}$, resulting in higher power consumption.

When comparing Arc 3 and Arc 1, it was noted that in Fig. 5 the equipment amount is greater for FTTH architecture in $\mathrm{HS}$ and $\mathrm{CO}$, but has less power consumption compared to FTTC, as shown in Fig. 3. The reason for this lies in the individual equipment energy consumption the in HS of the FTTH architecture. The splitters in HS do not consume energy because they are passive optical devices, which dramatically reduces the overall power consumption of the backhaul.

Furthermore, when analyzing the backhaul by segment, it was found that the higher energy consumption is in the "CS", as shown in Fig. 5. And, as noted in previous comparisons that switching devices for others with more backhaul efficient in the HS segment can dramatically reduce the total power consumption of the backhaul. It was tested an equipment type change in CS of the backhaul architecture, but for that it is necessary to change the type of BS. Therefore, it was chosen the FemtoWoC that has better performance and power consumption than FemtoC, as shown in [14]. As a result of this CS exchange, there was a significant reduction in total power consumption of the backhaul FTTB being less than the FTTH, as shown in Fig. 3. Despite the fact that FTTB backhaul for FemtoWoC and the FTTH for FemtoC having a similar structure when connection to CS, Fig. 4 shows that the equipment quantity in the three backhaul segments was lower for the FTTB. The reason for this lies in the FemtoWoC configuration that employs traffic aggregation and centralization of FemtoWoC BS in MCB. In other words, it is one McBS for $\mathrm{n}$ FemtoWoC, whereas in the FTTH the proportion is one ONU for each FemtoC, resulting in more equipment. Then, the ONUs set results in higher energy consumption compared to the McBS.

Therefore, the backhaul power consumption can be 
reduced by adopting architectures and equipment with better energy efficiency in its segments. And as the main bottleneck, the CS segment representing approximately $40 \%$ and $98 \%$ of the backhaul total energy consumption. However, it must important to pay attention to the HS for architectures that use copper technologies, such as XDL, because they consume a lot of energy.

\section{CONCLUSION}

It was presented in this research a new prediction of mobile traffic model for the next 15 years and an efficient base stations deploying model respecting the coverage area and the traffic requirements. Finally, it was studied the impacts that may be caused by the total energy consumption of the backhaul.

The backhaul was analyzed as a whole and its segments; Cell Site, Hub Site and Central Office. It was demonstrated how wireless network expansion, different architectures backhaul and capacity requirements affect the total energy consumption of the backhaul and its segments, as result determining bottlenecks.

The expected traffic boost implies increasing the infrastructure of all backhaul architectures impacting on the energy consumption. However, changing the backhaul architecture type can reduce the total energy consumption. Considering only FemtoC as indoor BS, it was found that the backhaul architectures FTTH and FTTB had the lowest and the highest energy consumption, respectively, due to the energy efficiency of the equipment in the "HS". However, it was also identified that the major bottleneck is the "CS", representing up to $98 \%$ of the whole energy consumption.

Thus, by changing to an indoor BS type more efficient, such as FemtoWoC, the equipment and the architecture of the "CS" was modified. Applying this change in the FTTB architecture, it was observed a significant improvement in the backhaul energy consumption achieving less power consumption than the FTTH with FemtoC. Therefore, adopting architectures and equipment with better energy efficiency we can reduce the backhaul power consumption.

\section{REFERENCES}

[1] P. Monti, S. Tombaz, L. Wosinska, and J. Zander, "Mobile backhaul in heterogeneous network deployments: Technology options and power consumption," in Proc. 2012 14th International Conference on Transparent Optical Networks (ICTON), July 2-5, 2012, pp. 1-7.

[2] S. Tombaz, P. Monti, K. Wang, A. Vastberg, M. Forzati, and J. Zander, "Impact of backhauling power consumption on the deployment of heterogeneous mobile networks," in Proc. 2011 IEEE Global Telecommunications Conference, Dec. 5-9, 2011, pp. 1-5.

[3] Ericsson Mobility Report: On the Pulse of the Networked Society, Ericsson, Stockholm, Sweden, November 2013.

[4] Ericsson Radio Dot System, Ericsson, Stockholm, Sweden, 2013.

[5] S. Tombaz, P. Monti, F. S. Farias, M. Fiorani, L. Wosinska, and J. Zander, "Is backhaul becoming a bottleneck for green wireless access networks?" in Proc. IEEE International Conference on Communications (ICC), Sydney, June 10-14, 2014, pp. 4029, 4035.
[6] I. E. Lee, Z. Ghassemlooy, W. P. Ng, and A. Khalighi, "Green-inspired hybrid FSO/RF wireless backhauling and basic access signalling for next generation metrozones," in Proc. 2012 2nd International Symposium on Environment Friendly Energies and Applications, June 25-27, 2012, pp. 230-236.

[7] Fiber, Microwave and Copper in the Mobile Backhaul - Business Case, 2nd Ed., SELTA Group, Milan, Italy, November 2010.

[8] Energy Efficiency Analysis of the Reference Systems, Areas of Improvements and Target Breakdown, Deliverable D2.3, EARTH project, January 2012.

[9] F. S. Farias, G. S. Borges, R. M. Rodrigues, A. L. Santana, and J. C. W. A. Costa, "Real-time noise identification in DSL systems using computational intelligence algorithms," in Proc. 2013 International Conference on Advanced Technologies for Communications (ATC), Oct. 16-18, 2013, pp. 252, 255.

[10] F. S. Farias et al., "Green backhauling for heterogeneous mobile access networks: What are the challenges?" in Proc. 2013 9th International Conference on Information, Communications and Signal Processing (ICICS), Dec. 10-13, 2013, pp. 1, 5.

[11] P. Vishwanath and D. Tse, Fundamentals of Wireless Communication, Cambridge University Press, 2005.

[12] Skatteverket and Sweco, City of Stockholm, SCB, SMHI, Stockholm, Sweden, 2013.

[13] Statistical Yearbook 2011, 56th issue, United Nations, New York, 2013.

[14] J. Gambini and U. Spagnolini, "Wireless over cable for femtocell systems," Communications Magazine, IEEE, vol. 51, no. 5, pp. 178-185, May 2013.

[15] H. Raza, "A brief survey of radio access network backhaul evolution: part I," Communications Magazine, IEEE, vol. 49, no. 6, pp. 164, 171, June 2011.

[16] W. Vereecken, W. V. Heddeghem, M. Deruyck, B. Puype, W. Joseph, D. Colle, L. Martens, and P. Demeester, "Power consumption in telecommunication networks: overview and reduction strategies," Communications Magazine, IEEE, vol. 49, no. 6, pp. 62, 69, June 2011.

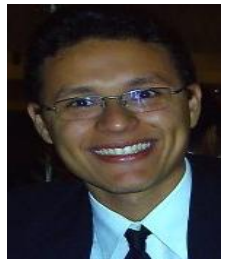

Albert Richard Moraes Lopes was born in Pará, Brazil on March 8, 1987. He received his bachelor's degree in computer engineering from the Universidade Federal do Pará (UFPA) in 2010. He is a researcher and one member of the LEA (Laboratory of Applied Electromagnetics) UFPA since 2012. His research interests include green network, mobile backhaul and heterogeneous networks.

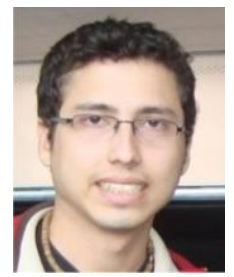

Fabrício S. Farias was born in Pará, Brazil on March 10, 1988. He received his bachelor's degree in computer engineering from the Universidade Federal do Pará (UFPA) in 2010. In 2011, he received his master's degree in eletrical engineering from UFPA Since 2010, he is a researcher and one member of the LEA (Laboratory of Applied Electromagnetics) UFPA. Also, he is an assistant professor at UFPA Campus Cametá since 2014. His research interests include data mining, DSL networks, mobile backhaul, digital TV and heterogeneous networks.

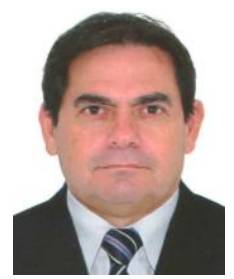

João Crisóstomo Weyl Albuquerque Costa was born in Pará, Brazil on January 27, 1959. He received his bachelor's degree in electrician/electronic engineering in 1981, and postgraduate degree in geophysics in 1983, both from UFPA. He received his master's degree in 1989 from PUC-RJ and PhD degree in electrical engineering in 1994 from UNICAMP-SP. Since 1994 he is a professor of electrical and computer engineering at UFPA. Currently, his areas of research are in modeling of optical devices and systems, including access networks. He is a researcher of CNPq since 1994 